\title{
Effects of Laser Illumination on Superconducting Circuits for Quantum Transduction
}

\author{
Srujan Meesala ${ }^{\mathrm{a}, *}$, Jash Banker ${ }^{\mathrm{a}}$, Steven Wood ${ }^{\mathrm{a}}$, Alp Sipahigil ${ }^{\mathrm{a}}$, David Lake ${ }^{\mathrm{a}}$, Piero \\ Chiappina $^{\mathrm{a}}$, Andrew Beyer ${ }^{\mathrm{b}}$, Matthew Shaw ${ }^{\mathrm{b}}$, Oskar Painter ${ }^{\mathrm{a}, \mathrm{c}}$ \\ a Thomas J. Watson, Sr., Laboratory of Applied Physics, California Institute of Technology, Pasadena, California \\ 91125, USA \\ ${ }^{\mathrm{b}}$ Jet Propulsion Laboratory, California Institute of Technology, Pasadena, California 91109, USA \\ ${ }^{\mathrm{c}}$ AWS Center for Quantum Computing, Pasadena, California 91125, USA \\ *srujan@caltech.edu
}

\begin{abstract}
Decoherence and noise from optical absorption in superconducting circuits hinder development of microwave to optical quantum transducers. Addressing these issues, we fabricate niobium-based resonators and qubits, and study them under laser illumination at milliKelvin temperatures. (C) 2021 The Author(s)
\end{abstract}

\section{Main Text}

Coherent conversion of quantum states between microwave (MW) and optical frequencies will allow the use of superconducting quantum processors in optical quantum networks. A variety of schemes being pursued to this end use a strong optical pump to mediate the nonlinear conversion process, and involve integration of a photonic device carrying relatively high optical power close to a superconducting circuit. Absorption of stray optical pump photons generates quasiparticles in the superconducting circuit, introducing excess decoherence and noise. This presents a major challenge for quantum transduction.
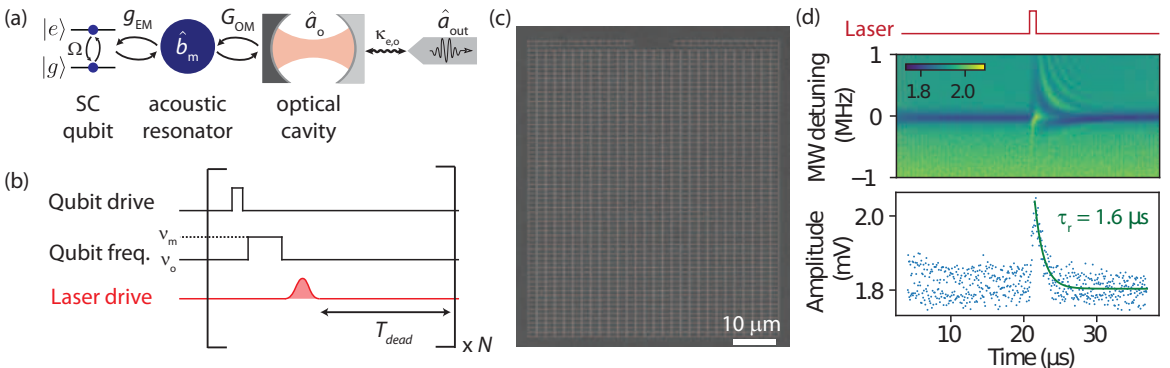

Fig. 1. (a) Schematic for transduction of a microwave state in a superconducting qubit to an optical photon using an acoustic mode as an intermediary. Various modes and interaction rates in the transducer are indicated. (b) Pulsed mode operation sequence of our transducer. Following qubit excitation via a MW drive at frequency $v_{\mathrm{o}}$, the qubit is brought into resonance with the acoustic mode at frequency $v_{\mathrm{m}}$ to swap the excitation into a phonon via a piezoelectric interaction. The phonon is then upconverted to an optical photon by a laser pulse sent to the optomechanical cavity. (c) Scanning electron microscope ( $\mathrm{SEM}$ ) image of frequency tunable $\mathrm{NbN}$ resonator. Light (dark) regions correspond to $\mathrm{NbN}(\mathrm{Si})$. (d) Recovery of $\mathrm{NbN}$ resonator upon illumination by a $1 \mu$ s long laser pulse (peak power: $500 \mu \mathrm{W}$ ) seen in its time-resolved spectrum (top panel). Exponential fit to response at zero detuning (bottom panel) yields a recovery time, $\tau_{r}=1.6 \mu \mathrm{s}$.

Recently, our group demonstrated a hybrid piezoelectric and optomechanical transducer that can generate optical photons from a superconducting qubit with signal-to-noise greater than unity [1]. Our scheme outlined in Fig. 1(a) uses phonons as intermediate excitations. To mitigate laser-induced noise and decoherence in the superconducting circuit, the transducer is operated in pulsed mode as shown in Fig. 1(b) with electrical and optical operations separated in time. Following the laser pulse, the transducer is inoperable for a time, $T_{\text {dead }} \sim 10 \mathrm{~ms}$ determined by quasiparticle relaxation in the aluminum (Al) transmon qubit. With a conversion efficiency of $10^{-5}$, this repetition rate yields an optical photon count rate of $1 \mathrm{mHz}$. An anti-bunching observation on upconverted 
photons is impractical with these parameters. Further, the qubit is unusable for remote entanglement generation after the laser pulse. As a solution to this challenge, we are pursuing integration of niobium-based circuits with our transducer. Fast quasiparticle relaxation in niobium $(\mathrm{Nb})$ and niobium nitride $(\mathrm{NbN})$ on the $\sim \mathrm{ns}$ timescale [2] could enable higher repetition rates. In this submission, we report laser illumination studies on two types of niobium-based superconducting devices.

Fig. 1(c) shows an SEM image of a frequency tunable $\lambda / 2$ resonator fabricated in thin film $(\sim 10 \mathrm{~nm}) \mathrm{NbN}$ on silicon-on-insulator (SOI). The resonator is shaped as a densely meandered ladder. In measurements that follow, samples are mounted on the mixing plate of a dilution refrigerator. A lensed fiber used to illuminate the device with $1.55 \mu \mathrm{m}$ laser light is positioned in the plane of the chip at distance of $\sim 1 \mathrm{~mm}$ from the circuit. This configuration is chosen to mimic scattering from end-fire coupling to the nanophotonic waveguide in our transducer geometry. We observe that the resonator spectrum recovers on a timescale $\tau_{r}=1.6 \mu$ s following a laser pulse as shown in Fig. $1(d)$. Set by the linewidth of our resonator, this timescale is an upper bound for the actual recovery process.
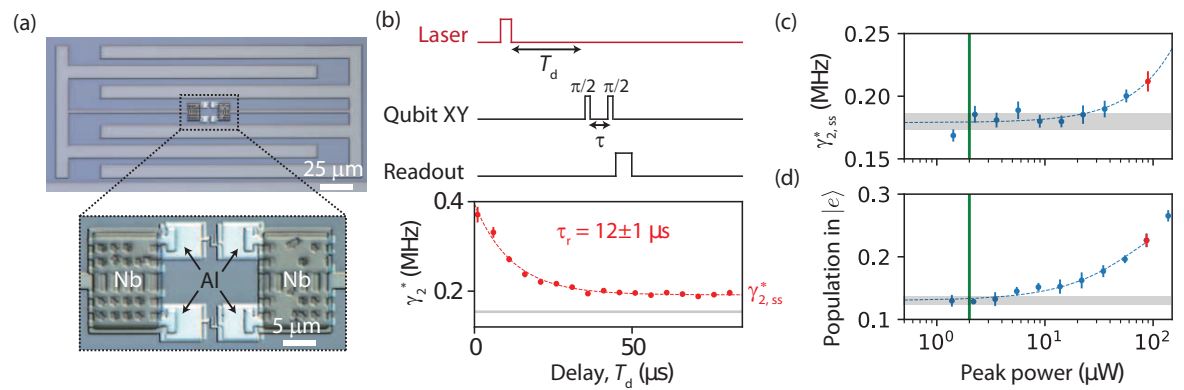

Fig. 2. (a) Optical image of $\mathrm{Nb}$ transmon qubit showing the $\mathrm{Nb}$ interdigitated capacitor and SQUID loop (shown at higher magnification in inset). (b) Recovery of decoherence rate of Nb qubit following a laser pulse. Top: Experimental pulse sequence repeated at $10 \mathrm{KHz}$ repetition rate. Bottom: Qubit decoherence rate, $\gamma_{2}^{*}$ versus delay, $T_{d}$ from a $100 \mathrm{~ns}$ long laser pulse (peak power: $90 \mu \mathrm{W}$ ). Gray line indicates decoherence rate when the laser is off. Dashed line is an exponential fit indicating a recovery time, $\tau_{r}=12 \pm 1 \mu \mathrm{s}$. With the laser on, the steady state decoherence, $\gamma_{2, s s}^{*}$ is higher than the laser off value. Dependence of (c) steady state decoherence, $\gamma_{2, s s}^{*}$ and (d) excited state population of the qubit as a function of peak laser power. Experimental sequence is repeated at $20 \mathrm{KHz}$ repetition rate. Horizontal gray regions indicate laser off values up to one standard deviation. Dashed line in (c) is a fit to the expression $\alpha+\beta P^{n}$, in (d) is a fit to $n_{\mathrm{th}}(\beta P)$, where $n_{\mathrm{th}}(\cdot)$ is the Bose-Einstein distribution. Vertical green line at $2 \mu \mathrm{W}$ corresponds to pump power level typical for our transducer.

Fig. 2(a) shows an optical image of the second device, a hybrid transmon qubit on Si formed by a Nb capacitor and a superconducting quantum interference device (SQUID) loop comprising conventional Al/AlOx/Al Josephson junctions. In the measurement shown in Fig. 2(b), we apply a Ramsey sequence at varying delay, $T_{d}$ from a laser pulse and observe fast recovery of qubit decoherence $\left(\gamma_{2}^{*}=1 / 2 \pi T_{2}^{*}\right)$ on the timescale $\tau_{r}=12 \pm 1 \mu \mathrm{s}$. The steady state decoherence, $\gamma_{2, s s}^{*}$ is higher than the laser-off value (horizontal gray line) due to a process slower than the repetition period $(100 \mu \mathrm{s})$. In Fig. 2(c), we plot the steady state decoherence, $\gamma_{2, s s}^{*}$ with varying laser power, and note that it follows a linear scaling $(n=1.0 \pm 0.2)$. We also probe qubit excited state population following the laser pulse, and observe a similar fast recovery followed by excess population due to a slow process. This steady state population is well described by a temperature that increases linearly with laser power as shown in Fig. 2(d), indicating that the slow process is likely of thermal origin. Importantly, at the typical pump power (few $\mu \mathrm{W}$, shown by vertical green line in Figs. 2(c,d)) used for optomechancial conversion, the qubit has a decoherence rate and an effective temperature identical to the laser-off situation (horizontal gray lines).

Measurements on both devices indicate that a repetition rate of $10 \mathrm{KHz}$, a 100x improvement over our previous work [1] is feasible. Combined with an efficiency boost to $10^{-3}$ from improvements to our optical setup, replacing the Al transmon in [1] with these niobium-based circuits will allow $10 \mathrm{~Hz}$ optical photon count rate. This will place experiments verifying the quantum coherent nature of our transduction scheme within reach.

\section{References}

1. M. Mirhosseini, A. Sipahigil, M. Kalaee, O. Painter, "Quantum transduction of optical photons from a superconducting qubit" arXiv:2004.04838 (2020)

2. P. J. De Visser, "Quasiparticle dynamics in aluminium superconducting microwave resonators", TU Delft (2014) 